Case Report

\title{
Effects of Pulmonary Rehabilitation on Respiratory Muscle Strength and Functional Capacity of Individuals with Chronic Obstructive Pulmonary Disease: Case Report
}

\author{
Nayara R. A. V. Boas ${ }^{1}$, Alessandra G. Cizino', Eloisa M. G. Regueiro ${ }^{4}$, Sueli A. Alves ${ }^{1}$, Edson D. \\ Verri $^{4}$, Gabriel P. Silva ${ }^{1,2}$, Oswaldo S. Taube ${ }^{1,2}$, Simone C. H. Regalo ${ }^{2}$, Saulo C. V. Fabrin ${ }^{*}, 1,2,3$ \\ ${ }^{1}$ University Center UNIFAFIBE, Bebedouro, São Paulo, Brazil. \\ ${ }^{2}$ Faculty of Dentistry of Ribeirão Preto - FORP/USP, Ribeirão Preto, São Paulo Brazil. \\ ${ }^{3}$ Faculty of Medicine of Ribeirão Preto - FMRP/USP, Ribeirão Preto, São Paulo, Brazil. \\ ${ }^{4}$ Claretiano University Center, Batatais, São Paulo, Brazil.
}

\begin{abstract}
Chronic obstructive pulmonary disease is a common preventable and treatable disease characterized by persistent airflow limitation that is usually progressive and associated with an enhanced chronic inflammatory response in the airways and the lung to noxious particles or gases. The objective of this study was to evaluate the effects of a pulmonary rehabilitation program on respiratory muscle strength and functional capacity of individuals with chronic obstructive pulmonary disease. An elderly male patient participated in the pulmonary rehabilitation program and was evaluated before and after intervention. This program consisted of four phases: stretching, aerobic exercises, resistance training and relaxation. The physical activities were provided three times a week on alternate days, for 4 months, totaling 48 sessions. The results obtained after the rehabilitation program showed improvement in the physical functional capacity of the patient measured by the six-minute walk test $($ Pre $=480 ;$ Post $=540 \mathrm{~m})$. The maximal inspiratory pressure values increased $\left(P r e=-60 ;\right.$ Post $\left.=-120 \mathrm{cmH}_{2} \mathrm{O}\right)$ whereas the maximal expiratory pressure values remained the same $(P r e=50$; Post $=50 \mathrm{cmH}_{2} \mathrm{O}$ ). Therefore, the respiratory rehabilitation program has improved functional capacity and inspiratory muscle strength.
\end{abstract}

Key words: COPD, physical exercise, cardiorespiratory fitness, walk test, dyspnea.

\section{Introduction}

Chronic obstructive pulmonary disease (COPD) is one of the leading causes of death worldwide and it is estimated that it will become the third by 2030 . It is characterized by persistent airflow limitation that is usually progressive and is caused by an enhanced chronic inflammatory response in the airways and the lung to noxious particles or gases and is often associated with smoking. 1,2

COPD exacerbations have been defined as a change in baseline dyspnea, cough and/or sputum. These adverse events are also related to the loss of functional capacity and subsequent physical dependence in basic and instrumental activities of daily living. ${ }^{3}$ Therefore, the prescription of controlled physical exercises can activate the mechanisms that provide increased exercise tolerance, relief of dyspnea, and improve the quality of life and functional capacity. ${ }^{4,5}$

The rehabilitation process is essential for COPD patients since it consists of an individualized treatment to alleviate symptoms, optimize functional capacity, maintaining the maximum level of activity, and stabilizing or reversing the systemic manifestations of the disease. ${ }^{6}$ Pulmonary rehabilitation is an educational and exercise program aiming to control the respiratory deficit of individuals, increase endurance, reduce dyspnea and prevent musculoskeletal changes. $^{7}$
The effects of pulmonary rehabilitation on COPD patients can be measured by the six-minute walking test (6MWT), which is used to assess the functional capacity of non-trained individuals and those who are not able to perform the cardiac stress test. ${ }^{8}$ It is the most frequently used exercise capacity assessment for patients with chronic lung disease and is also helpful in therapeutic interventions. ${ }^{9}$ These interventions influence the respiratory mechanisms and their effects can be measured using manovacuometry, which is a complementary test to assess the respiratory muscle strength. The manovacuometer is reliable equipment in which the maximal inspiratory pressure and the maximal expiratory pressure are obtained. $^{10}$

Therefore, the objective of this study was to evaluate the effects of a pulmonary rehabilitation program on respiratory muscle strength and functional capacity of individuals with chronic obstructive pulmonary disease.

\section{METHODS}

\section{Sample characteristics}

The present study was characterized as a case report. The assessment of the functional capacity and the respiratory muscle strength of a male patient with a clinical diagnosis of COPD was conducted at the Clínica Escola de Fisioterapia do 
UNIFAFIBE, São Paulo, Brazil. Assistance was provided by physiotherapy students.

A 63-year-old sedentary male (height of $1.78 \mathrm{~cm}$, weight of 90 $\mathrm{kg}$, and BMI of $28.4 \mathrm{~kg} / \mathrm{m} 2$ ) participated in the rehabilitation program. The patient has not been smoking and drinking for 22 years and was diagnosed with COPD 21 years ago, with signs and symptoms of pulmonary emphysema.

The subject signed a written informed consent previously approved by the local Research Ethics Committee (under N. 2.103.67), according to the Brazilian National Health Council (resolution N. 466/12). The patient was duly informed about the rights and obligations, risks and benefits of the procedures and was free to leave the study at any time, without any consequences concerning present or future activities.

\section{Data collection}

The clinical evaluation was comprised of anamnesis, anthropometric measures, respiratory muscle strength and sixminute walk test data. All pre- and post-exercise testing and experimental procedures were performed by the same physical therapist, at the same time of day, following the same protocol. Anamnesis and the respiratory muscle strength data were collected on the same day; the other measures were obtained on alternate days in order to avoid fatigue and dyspnoea, which are common symptoms in COPD individuals. Height and body mass (BM) were measured (Welmy 110FF, São Paulo, SP, Brazil) with the subject in a standing position, looking forward and barefoot. Commonly accepted BMI ranges are underweight: under $18.5 \mathrm{~kg} / \mathrm{m} 2$, normal weight: 18.5 to 25 , overweight: 25 to 30 , obese: over $30 .^{11}$

The 6MWT was performed in a 30-m corridor with the use of a portable oximeter (Nonin Medical 2500, Inc., Plymouth, MN, USA), in accordance with the recommendations of the American Thoracic Society. ${ }^{12}$ The patient was instructed to walk as fast as he could for six minutes, with no monitoring or talking during the test, except to report any symptoms or difficulties while performing the test. Every minute, the individual was verbally encouraged to keep walking with phrases such as: "you're doing really well!", "Three minutes to complete the test." Every two minutes, the peripheral capillary oxygen saturation $\left(\mathrm{SpO}_{2}\right)$ was verified with the use of a portable pulse oximeter (Nonin Medical 2500, Inc., Plymouth, MN, USA), heart rate (HR, Polar Vantage NVTM, Model 1901001, Kempele, Oulu, Finland), symptoms of dyspnea and respiratory rate were verified by the Borg modified scale CR10. ${ }^{13}$ Blood pressure (BP) was measured at rest and immediately after testing.

Respiratory muscle strength was assessed to measure the maximum inspiratory (MIP) and maximal expiratory (MEP) pressures, according to the procedure used by Black and Hyatt. $^{14}$ Both pressure levels were measured with a manometer graduated in $\mathrm{cmH}_{2} \mathrm{O}$ and equipped with a mouthpiece (Ger-Ar®, São Paulo, Brazil). The maneuver was performed with the patient in a sitting position, with a nose clip, knees and elbows flexed at $90^{\circ}$. MIP was measured at the mouth after complete exhalation to residual volume (RV) followed by a single sustained maximal inspiratory effort from that lung volume against an occluded airway. For the MEP measurement, the patient inhaled up to the total lung capacity. Both pressures were sustained for at least one second. Three to five maneuvers were conducted with 1-minute interval between the measurements, with a variation less than or equal to $10 \%$ of the greatest value. The greatest value was selected for analysis before and after the rehabilitation protocol, in accordance with that described by Neder et al. (1999). ${ }^{15}$

\section{Pulmonary rehabilitation program}

The pulmonary rehabilitation program (PRP) started on May10, 2017 and was carried out for four months, three times a week, on alternate days, totaling 48 sessions. After this period, the individual was evaluated again with the same initial tests on August 22, 2017. At the beginning and at the end of the sessions, $\mathrm{BP}, \mathrm{SpO}_{2}, \mathrm{HR}$, Borg and pulmonary auscultation were verified, however, $\mathrm{HR}, \mathrm{SpO}_{2}$ and $\mathrm{BP}$ were also measured during the sessions for monitoring purposes.

The PRP was divided into four steps: stretching, aerobic physical training on a treadmill, upper and lower limbs resistance training and relaxation.

1st step: Stretching - cervical stretching of the upper and lower limbs was performed at the beginning of the sessions, for 10 minutes, bilaterally and 20 seconds for each movement. The exercises focused on the following muscle groups: sternocleidomastoid, scalene, pectoralis major, teres major, triceps, subscapularis, wrist flexors and extensors, quadriceps, hamstrings, gastrocnemius and soleus.

2nd step: Aerobic physical training on a treadmill - the cardiorespiratory workout was performed on both a stationary bike and a treadmill, alternating between the two. The bike workout was conducted with a cycling power of 1 watt. The treadmill workout was performed with zero inclination at max speed of $4 \mathrm{~km} / \mathrm{h}$. In the course of the study, the initial time for the treadmill and the bike workouts was 20 minutes and was later increased to 40 minutes.

The aerobic training started between 60 to $70 \%$ of maximum heart rate (HRmax) measured by the $6 \mathrm{MWT},{ }^{16}$ with weekly adjustment of the training load, using the rates of perceived dyspnea sensation on a CR10BORG scale between 4-6 of $10,{ }^{17}$ with the HR determined for each training period, with the possibility of reaching up to $85 \%$ HRmax during rehabilitation. Supplemental oxygen was not necessary during training.

3rd step: Resistance training - It was carried at the weight room and focused on the following lower limb muscles: hip flexors, extensors, adductors and abductors; knee flexors and extensors. The upper limb muscles included the shoulder flexors, extensors, adductors, and abductors; the elbow flexors and extensors. The initial load was set at 50\% 1RM with increases of $10 \%$ every 3 weeks. Three sets of 12 repetitions, evolving to 15 repetitions with a two-minute interval between the series. 
4th step: Relaxation - the sessions were followed by 5-minute relaxation exercises, which consisted of diaphragmatic breathing, diaphragmatic breathing (3 times) and diaphragmatic breathing during upper limb elevation.

\section{RESULTS}

After the rehabilitation protocol, the results were processed and analyzed comparatively. There was improvement in the vital signs at rest and the walking distance increased during the 6MWT, as shown in Table1.

Table 1 - Comparison of pre- and post-pulmonary rehabilitation values

\section{MWT Pre-rehabilitation Post-Rehabilitation}

\begin{tabular}{l|cc}
\hline HR & $90 \mathrm{bpm}$ & $64 \mathrm{bpm}$ \\
RR & $19 \mathrm{ipm}$ & $17 \mathrm{ipm}$ \\
BP & $120 \times 80 \mathrm{mmHg}$ & $110 \times 70 \mathrm{mmHg}$ \\
SpO $_{2}$ & $90 \%$ & $97 \%$ \\
BORG & 0 & 0 \\
DC & 480 meters & 540 meters \\
\hline
\end{tabular}

Heart rate (HR), respiratory rate (RR), blood pressure (BP), blood oxygen saturation $\left(\mathrm{SpO}_{2}\right)$, Borg scale (BORG), distance covered in meters (DC).

The comparison between the pre- and post-rehabilitation values through manovacuometry revealed improvement for MIP whereas those for MEP were similar, as shown in Table 2.

Table 2 - Comparison of respiratory muscle strength preand post-rehabilitation

Manovacuometry

Prerehabilitation

MIP
- $60 \mathrm{cmH}_{2} \mathrm{O}$ MEP $+50 \mathrm{cmH}_{2} \mathrm{O}$ $+50 \mathrm{cmH}_{2} \mathrm{O}$

Maximal inspiratory pressure (MIP) and maximal expiratory (MEP) expressed in $\mathrm{cmH}_{2} \mathrm{O}$.

\section{DISCUSSION}

The results obtained showed an improvement in the functional capacity of the individual since there was an increase in the distance covered during the 6MWT, following the pulmonary rehabilitation program. The subject covered a 480 meter distance pre-rehabilitation and a greater distance (540 meters) post-rehabilitation. Rodrigues et al. $^{18}$ reported that 12 individuals performed the 6MWT and the comparison between the pre- and post-pulmonary rehabilitation values revealed improvement in the distance covered from 391 to 442 meters, respectively.

According to the study of Coquart et al. ${ }^{19}, 35$ outpatients with COPD performed the 6MWT before and after pulmonary rehabilitation. The comparative results showed that the distance covered significantly increased from 514 to 559 meters.

Our findings corroborate those reported by Costa et al. ${ }^{20}$ The authors evaluated 78 COPD patients using the 6MWT6 preand post-pulmonary rehabilitation. A significant increase was observed in the distance covered (375 to 420 meters). In another study, Fabrin et al. ${ }^{21}$ evaluated a COPD patient and found a significant improvement in the distance covered (345 to 490 meters) when the values of a 5-month pre-and postcardiopulmonary rehabilitation values were compared.

The assessment of the respiratory muscle strength through manovacuometry showed improvement of MIP $\left(-60 \mathrm{cmH}_{2} \mathrm{O}\right.$ pre-intervention, $-120 \mathrm{cmH}_{2} \mathrm{O}$ post-intervention) while the MEP values remained the same. Zanchet et al. ${ }^{22}$ demonstrated an increase of MIP $\left(-89 \mathrm{cmH}_{2} \mathrm{O}\right.$ pre-intervention, $-102 \mathrm{cmH}_{2} \mathrm{O}$ post-intevention). The authors suggested that the improvement of inspiratory muscle strength was due to the overall fitness presented by the individual and not by specific respiratory muscle training.

Trevisan et al. ${ }^{23}$ reported that changes in MEP were barely noticeable in COPD patients. This finding is consistent with that found in the present study i.e. similar MEP values obtained after the pulmonary rehabilitation program. Kunikoshita et al. ${ }^{24}$ conducted a study with 25 COPD patients, who were divided into 3 groups: Group1, submitted to respiratory muscle training; Group 2, submitted to physical training; and Group 3, submitted to respiratory muscle training and physical training for 6 weeks. Group 1 and 3 showed improvement in MIP (-49 to $-57,-51$ to $-77 \mathrm{cmH}_{2} \mathrm{O}$, respectively), suggesting that this improvement has occurred due to specific training for respiratory muscles and not to physical training, which does not corroborate the present study. Therefore, these interesting variables should be analyzed in further studies in order to direct the breathing exercises and their correlation with the improvement in respiratory muscle strength (MIP and MEP).

\section{CONCLUSION}

The results obtained showed that the pulmonary rehabilitation program had beneficial effects on the COPD patient since it has improved the functional capacity and increased the inspiratory muscle strength.

\section{REFERENCES}

[1] Fabbri LM, Hurd SS. Global strategy for the Diagnosis, management and prevention of COPD: 2003 update. Eur Respir J. 2006; 22:1-2.

[2] Global Initiative for Chronic Obstructive Lung Disease (GOLD) Global Strategy for Diagnosis, management, and prevention of Chronic Obstructive Pulmonary Disease (Update 2017). Available at: <http://www.goldcopd.org/guidelines-global-strategy-fordiagnosis-management.html>. Accessed in: 20/03/2017.

[3] Cooper CB. Airflow obstruction and exercise. Respiratory Medicine. 2009; 103:325-34.

[4] Nascimento O, Iamonti V, Jardim J. Pulmonary Rehabilitation. SOPTERJ. Sociedade de Pneumologia e Tisiologia do Estado do Rio de Janeiro, Pulmão. 2013;22(2):65-69.

[5] Machado MG. Bases da fisioterapia respiratória - Terapia intensiva e reabilitação, Guanabara Koogan, Rio de Janeiro. 2016;458-468. 
Saulo C. V. Fabrin et al / Effects of Pulmonary Rehabilitation on Respiratory Muscle Strength and Functional Capacity of Individuals with Chronic Obstructive Pulmonary Disease: Case Report

[6] Sousa JBF, Ruas G, Volpe MS. Effects of a standard pulmonary rehabilitation program after a minimal period of treatment. Revista Brasileira Ciência. e Movimento. 2014;22(3):126-132.

[7] American Thoracic Society. Pulmonary rehabilitation. American Thoracic Society Patient Information Series. Am J Respir Crit Care Med. 2013; 188:5- 6.

[8] Pires SR, Oliveira AC, Parreira VF. Six-minute walk test at different ages and body mass indexes. Revista Brasileira de Fisioterapia. 2007;11(2):147-151.

[9] Britto RR, Sousa LAP. Six Minute Walk Test - a Brazilian Standardization. Fisioterapia em Movimento. 2006:19(4):49-54

[10] Bessa EJC, Lopes AJ, Rufino R. The importance of measurement of respiratory muscle strenght in pulmonology practice. Pulmão. 2015;24(1):37- 41 .

[11] Prescott E, Almdal T, Mikkelsenz KL, Tofteng CL, Vestbo J, Lange P. Prognostic value of weight change in chronic obstructive pulmonary disease: results from the Copenhagen City Heart Study. Eur Respir J. 2002; 20:539- 544.

[12] American Thoracic Society Statement: Guidelines for the Six-Minute Walk Test. Am J Respir Crit Care Med. 2002; 166:111-17.

[13] Borg GAV. Psysichophysical bases of perceived exertion. Med Sci Sport Exerc. 1982; 14:377-81.

[14] Black LF, Hyatt RE. Maximal respiratory pressures: normal values and relationship to age and sex. Am Rev Respir Dis.1969;99(5):696-702.

[15] Neder JA, Andreoni S, Lerario MC, Nery LE. Reference values for lung function tests. II. Maximal respiratory pressures and voluntary ventilation. Braz J Med Biol Res. $1999 ; 32: 719-727$.

[16] Dourado VZ, Tanni SE, Antunes LCO, Paiva SAR, Campana AO, Renno ACM, Godoy I. Effect of three exercise programs on patients with chronic obstructive pulmonary disease. Braz J Med Biol Res. 2009;42(3):263271.

[17] Langer D, Probst VS, Pitta F, Burtin C, Hendriks E, Schans CPVD, Paterson WJ, et al. Clinical Practice Guideline for physical therapy in patients with Chronic Obstructive Pulmonary Disease (COPD) - Portuguese version. Rev Bras Fisioter. 2009; 13:183-204.

[18] Rodrigues C, Alves L, Matsuo T, Gonçalves C, Hayashi D. The effect of an exercise program directed to the thoracic mobility in COPD. Fisioterapia. Mov. 2012; 25(2):343-349.

[19] Coquart J, Lemaitre F, Castres I, Saison S, Bart F, Grosbois J. Reproducibility and Sensitivity of the 6Minute Stepper Test in Patients with COPD.COPD. Journal of Chronic Obstrutive Pulmonary Disease.2015;12(5):533-538.

[20] Costa CC, Baldessar LZ, Canterle DB, Moussalle LD, Vettorazzi SF, Lermen CA, Teixeira PJZ. Analysis of results of a pulmonary rehabilitation program in patients with chronic obstructive pulmonary disease. Revista da AMRIGS. 2010;54(4):406-410.
[21] Fabrin S, Soares N, Regalo SCH, Vianna JRF, Regueiro EMG. Evolution of patients with heart disease after cardiopulmonary rehabilitation program: case report. Fisioter. Mov. 2017; 30(1):169-76.

[22] Zanchet RC, Vegas CAA, Lima T. Efficacy of pulmonary rehabilitation: exercise capacity, respiratory muscle strength and quality of life in patients with chronic obstructive pulmonary disease. Jornal Brasileiro de Pneumologia. 2005;30(2):118-24.

[23] Trevisan ME, Porto AS, Pinheiro TM. Influence of respiratory and lower limb muscle training on functional performance of subjects with COPD. Fisioterapia e Pesquisa. 2010;17(3):209-13.

[24] Kunikoshita LN, Silva YP, Silva TLP, Costa D, Jamami M. Effects of three respiratory physical therapy programs on patients with COPD. Rev. bras. fisioter. 2006;10(4):449-455. 